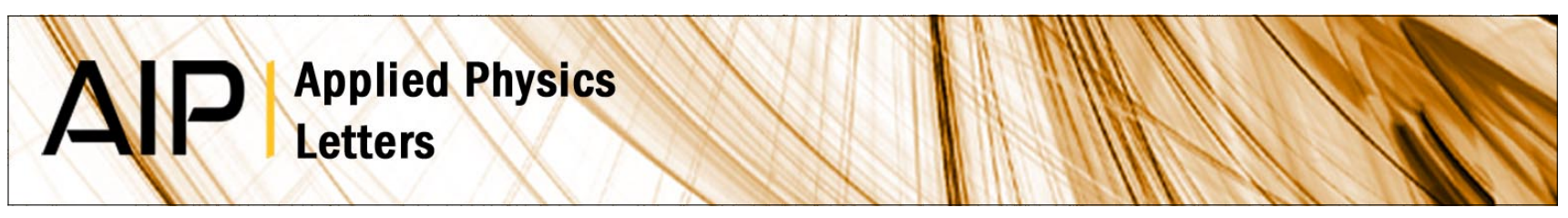

\title{
Energetic stability and magnetic properties of Mn dimers in silicon
}

F. Bernardini, S. Picozzi, and A. Continenza

Citation: Appl. Phys. Lett. 84, 2289 (2004); doi: 10.1063/1.1688002

View online: http://dx.doi.org/10.1063/1.1688002

View Table of Contents: http://apl.aip.org/resource/1/APPLAB/v84/i13

Published by the American Institute of Physics.

\section{Related Articles}

Point defect engineering strategies to suppress A-center formation in silicon

Appl. Phys. Lett. 99, 241901 (2011)

Spectroscopic studies of iron and chromium in germanium

J. Appl. Phys. 110, 113707 (2011)

Photoluminescence of deep defects involving transition metals in Si: New insights from highly enriched 28Si App. Phys. Rev. 2011, 15 (2011)

Photoluminescence of deep defects involving transition metals in Si: New insights from highly enriched 28Si J. Appl. Phys. 110, 081301 (2011)

Improved calculation of vacancy properties in Ge using the Heyd-Scuseria-Ernzerhof range-separated hybrid functional

J. Appl. Phys. 110, 063534 (2011)

\section{Additional information on Appl. Phys. Lett.}

Journal Homepage: http://apl.aip.org/

Journal Information: http://apl.aip.org/about/about_the_journal

Top downloads: http://apl.aip.org/features/most_downloaded

Information for Authors: http://apl.aip.org/authors

\section{ADVERTISEMENT}

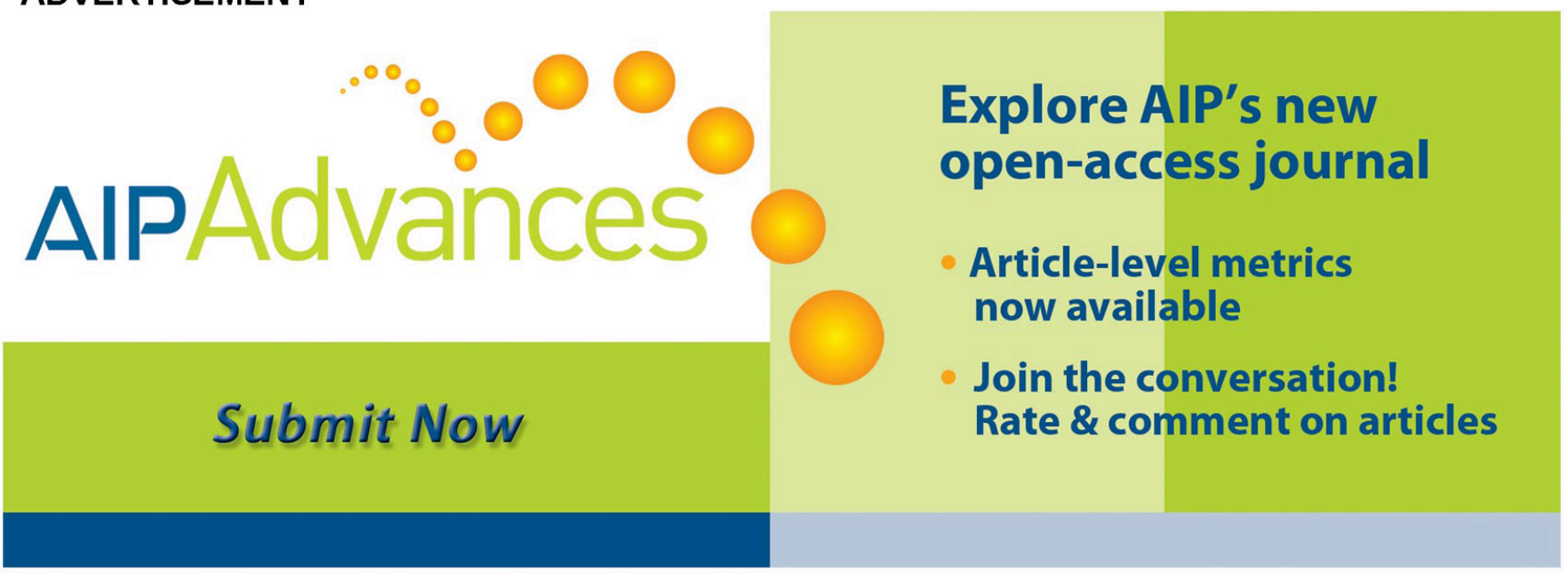




\title{
Energetic stability and magnetic properties of Mn dimers in silicon
}

\author{
F. Bernardini ${ }^{\text {a) }}$ \\ INFM-SLACS Sardinian Laboratory for Computational Materials Science and Dipartimento di Fisica, \\ Università degli Studi di Cagliari, 09042 Cagliari, Italy \\ S. Picozzi and A. Continenza \\ Istituto Nazionale di Fisica della Materia (INFM) and Dipartimento di Fisica, Università degli Studi di \\ L'Aquila, I-67010 Coppito (L'Aquila), Italy
}

(Received 11 November 2003; accepted 23 January 2004)

\begin{abstract}
We present an accurate first-principles study of magnetism and energetics of single Mn impurities and $\mathrm{Mn}$ dimers in Si. Our results, in general agreement with available experiments, show that (i) Mn atoms tend to aggregate, the formation energy of dimers being lower than the sum of the separate constituents, (ii) ferromagnetic coupling is favored between the Mn atoms constituting the dimers in $p$-type $\mathrm{Si}$, switching to an antiferromagnetic coupling in $n$-type $\mathrm{Si}$, (iii) $\mathrm{Mn}$ atoms show donors (acceptor) properties in $p$-type ( $n$-type) $\mathrm{Si}$, therefore they tend to compensate doping, while dimers being neutral or acceptors allow for Si to be doped $p$-type. (C) 2004 American Institute of Physics. [DOI: $10.1063 / 1.1688002]$
\end{abstract}

The discovery of ferromagnetism in diluted magnetic semiconductors ${ }^{1}$ - a new class of materials having GaMnAs as a prototypical compound-has renewed the interest in the physics of transition metal (TM) atoms in semiconductors. While most of the early works were focused on the TM doping of III-V and II-VI ${ }^{2}$ semiconductors, recently, ferromagnetic properties and magnetoresistance phenomena below room temperature have been reported for $\mathrm{Mn}_{x} \mathrm{Ge}_{1-x}$ ( $x$ up to $3 \%$ ). ${ }^{3}$ This discovery opened the issue of ferromagnetism in Mn-doped group-IV semiconductors. It is well known that TM solubility in Si and Ge is very low and that $\mathrm{Mn}$ atoms tend to aggregate into clusters. Electronparamagnetic resonance $(\mathrm{EPR})^{4}$ investigations showed that isolated $\mathrm{Mn}$ impurities in $\mathrm{Si}$ can occur in different charge states, both at interstitial $\left(\mathrm{Mn}_{\mathrm{I}}^{-}, \mathrm{Mn}_{\mathrm{I}}^{0}, \mathrm{Mn}_{\mathrm{I}}^{+}\right.$, and $\left.\mathrm{Mn}_{\mathrm{I}}^{2+}\right)$ and substitutional $\left(\mathrm{Mn}_{\mathrm{Si}}^{2-}\right.$ and $\left.\mathrm{Mn}_{\mathrm{Si}}^{+}\right)$sites.

From the theoretical point of view, pioneering firstprinciples Green's-function simulations were performed for isolated impurities in $\mathrm{Si}$, taking into account spin multiplicities and charge states. ${ }^{5,6}$ However, a careful theoretical study focused on the interaction between Mn atoms in a Si matrix based on large supercell density functional calculations including structural relaxation, is still lacking. A thorough investigation of Mn clustering in $\mathrm{Si}$ and $\mathrm{Ge}$ is outside the scope of this work and, in general, of first principles investigations. Moreover, technical issues related to the well-known underestimation of the band gap width in the framework of density functional theory (DFT) (Ge is depicted as a nearly zero gap material) suggested limiting the present investigation to $\mathrm{Mn}$ in silicon. Therefore, in this work we focus our attention on the energetics and on the magnetic properties of $\mathrm{Mn}$ isolated impurities and dimers in the limit of low doping concentrations $(\sim 0.5 \%-1 \%)$.

We compute the formation energies for the defects studied using the standard formalism by Zhang and Northrup. ${ }^{7}$

\footnotetext{
a) Author to whom correspondence should be addressed; electronic mail: fabio.bernardini@dsf.unica.it
}

Total energies and chemical potentials are computed within the DFT and the generalized gradient approximation of Perdew and Wang $^{8}$ to the exchange-correlation functional, using the projector augmented wave $\operatorname{method}^{9}$ and the plane-waves code provided in the VASP package. ${ }^{10}$ Spin polarization of valence electrons is treated semirelativistically (i.e., without spin-orbit coupling). As for the chemical potential of bulk $\mathrm{Mn}$, we use its calculated value in the antiferromagnetic (AFM) phase of its fcc metallic structure, while for silicon we refer to the equilibrium diamondlike structure. We simulate an isolated defect within the periodic boundary conditions via the repeated supercell approach, using a $3 \times 3 \times 3$ cubic supercell. Test calculations show that this is the smaller supercell dimension necessary to get converged results for the magnetic moments of the dimer complexes considered in this work. A kinetic energy cutoff of $202 \mathrm{eV}$ is used in all of the calculations. The supercell size and shape are kept fixed during the calculations. To minimize the effect of the interaction among the periodic images we use the $\mathbf{k}$ point set proposed by Makov, Shah, and Payne. ${ }^{11}$

We investigated the most important defects involving up to two Mn atoms: (i) tetrahedral interstitial $\mathrm{Mn}_{\mathrm{I}}$, (ii) $\mathrm{Mn}$ substituting $\mathrm{Si}\left(\mathrm{Mn}_{\mathrm{Si}}\right)$, (iii) a $\mathrm{Mn}$ dimer $\left(\mathrm{Mn}_{\mathrm{Si}}-\mathrm{Mn}_{\mathrm{Si}}\right)$ where two $\mathrm{Mn}_{\mathrm{Si}}$ sit on neighboring sites, (iv) a complex $\left(\mathrm{Mn}_{\mathrm{Si}}-\mathrm{Mn}_{\mathrm{I}}\right)$ formed by a $\mathrm{Mn}_{\mathrm{I}}$ bound to a $\mathrm{Mn}_{\mathrm{Si}}$, (v) an interstitial dimer $\left(\mathrm{Mn}_{\mathrm{I}}-\mathrm{Mn}_{\mathrm{I}}\right)$ where both $\mathrm{Mn}$ atoms occupy tetrahedral interstitial sites, (vi) a complex $\left(\mathrm{Mn}_{\mathrm{Si}^{-}}-\mathrm{Si}_{\mathrm{I}}\right)$ where an interstitial silicon atom $\mathrm{Si}_{\mathrm{I}}$ is bound to a $\mathrm{Mn}_{\mathrm{Si}}$. We performed a structural optimization allowing only lattice distortions compatible with $C_{3 v}$ symmetry. In Fig. 1 we show the formation energies for the defects involving a single $\mathrm{Mn}$ atom, i.e., $\mathrm{Mn}_{\mathrm{Si}}, \mathrm{Mn}_{\mathrm{I}}$, and the $\mathrm{Mn}_{\mathrm{Si}^{-}} \mathrm{Si}_{\mathrm{I}}$ complex. We find that, for isolated $\mathrm{Mn}$ atoms, interstitial atoms are energetically favored over substitutional ones for most of the allowed range of the electron chemical potential, in agreement with experimental evidence. ${ }^{12}$ The formation energy difference between $\mathrm{Mn}_{\mathrm{Si}}$ and $\mathrm{Mn}_{\mathrm{I}}$ is larger in $p$-type conditions and decreases for increasing Fermi level positions. In $n$-type conditions $\mathrm{Mn}_{\mathrm{Si}}$ 


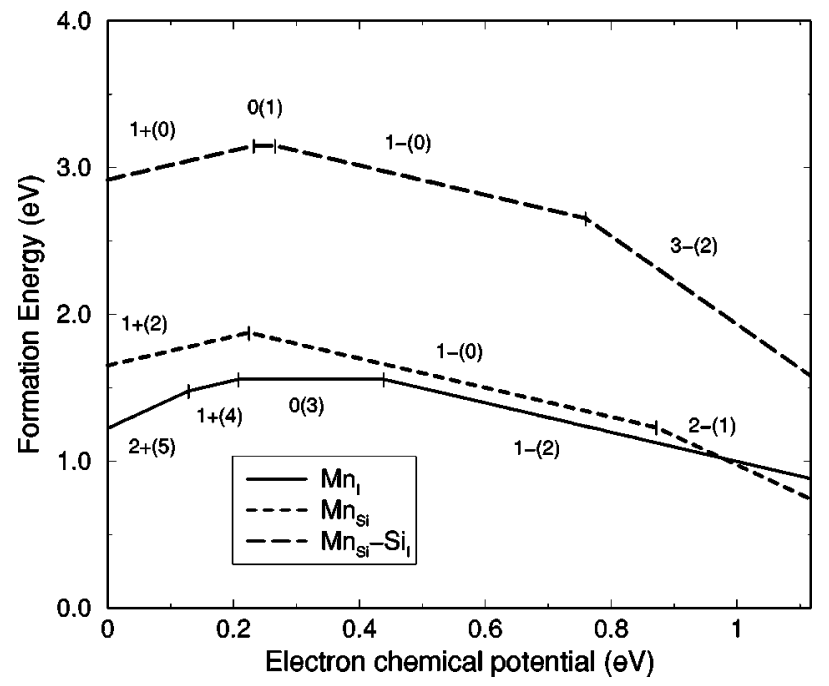

FIG. 1. Formation energy of single Mn impurities as a function of the Fermi level. Charge states are shown, along with the relative total magnetic moments, in Bohr magnetons (number in parentheses). To help readability, small vertical bars mark the ionization levels.

becomes a-double acceptor and for $E_{F}$ above $0.97 \mathrm{eV}$ it becomes energetically favorite over $\mathrm{Mn}_{\mathrm{I}}$. Therefore, we predict, in $n$-type $\mathrm{Si}$, the coexistence of $\mathrm{Mn}_{\mathrm{I}}$ and $\mathrm{Mn}_{\mathrm{Si}}$. As for the charge states of the defects, experimental data available in literature ${ }^{4}$ show the existence of charge states from $1-$ to $2+$ for the interstitial impurity, while for the substitutional one the only charge states detected are the $1+$ and $2-$. Besides a general agreement with experiments, our calculations reveal for the $\mathrm{Mn}_{\mathrm{Si}}$ the possible existence of a 1- charge state that was not reported in literature. The latter corresponds to a nonmagnetic configuration for the impurity, whose existence cannot be detected by EPR spectroscopy.

The incorporation of $\mathrm{Mn}$ in silicon can be achieved during crystal growth or by ion implantation. The second approach induces in the host crystal a large number of defects, that have to be eliminated by thermal annealing. During the annealing, two processes can occur: (i) a recombination of $\mathrm{Mn}_{\mathrm{I}}$ with silicon vacancies that favors the incorporation of $\mathrm{Mn}$ in the substitutional site and (ii) a "kick-out" process, where a substitutional $\mathrm{Si}$ is displaced by an interstitial $\mathrm{Mn}$ to a neighboring interstitial site. The final result of the latter is a $\mathrm{Mn}_{\mathrm{Si}^{-}} \mathrm{Si}_{\mathrm{I}}$ complex whose energy is plotted in Fig. 1. Our results clearly show that the $\mathrm{Mn}_{\mathrm{Si}^{-}}-\mathrm{Si}_{\mathrm{I}}$ complex is strongly energetically unfavored. Therefore, we conclude that kickout mechanisms are unlikely and most of the implanted Mn atoms will remain interstitial.

We now turn our attention to defects formed by two Mn atoms. In realistic conditions, high doping levels are achieved out of thermodynamical equilibrium. At these high concentrations, the pairing of Mn atoms is likely. In Fig. 2 the formation energy per Mn atom of the most important complexes involving Mn pairs is shown. The lowest energy configuration among those studied are $\mathrm{Mn}_{\mathrm{Si}}-\mathrm{Mn}_{\mathrm{I}}$ for $E_{F}$ up to $0.6 \mathrm{eV}$ and the double substitutional $\mathrm{Mn}_{\mathrm{Si}}-\mathrm{Mn}_{\mathrm{Si}}$ in $n$-type material, whereas the double interstitial $\mathrm{Mn}_{\mathrm{I}}-\mathrm{Mn}_{\mathrm{I}}$ is always too high in energy to be an energetically stable configuration. A noticeable feature of Fig. 2 is that formation energies for $\mathrm{Mn}_{\mathrm{Si}}-\mathrm{Mn}_{\mathrm{I}}$ and $\mathrm{Mn}_{\mathrm{Si}}-\mathrm{Mn}_{\mathrm{Si}}$ complexes are lower in energy than the sum of the formation energies for their individual

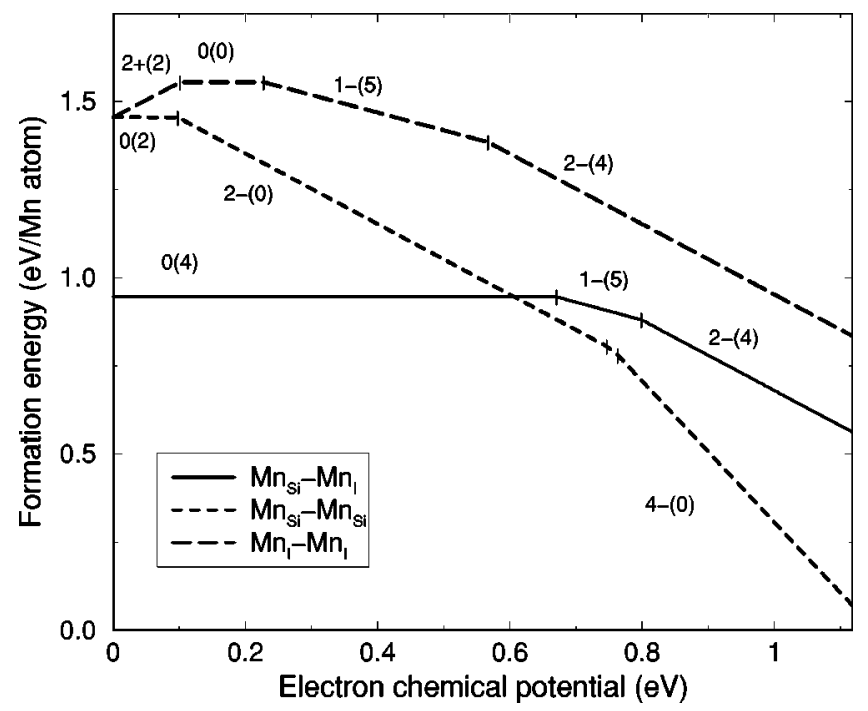

FIG. 2. Formation energy of Mn dimers as a function of the Fermi level, in unit of eV/Mn atom. Charge states are shown, along with the relative total magnetic moments, in bohr magnetons (number in parentheses).

components (i.e., $\mathrm{Mn}_{\mathrm{Si}}$ and $\mathrm{Mn}_{\mathrm{I}}$ ), meaning that an attractive interaction between $\mathrm{Mn}$ atoms exists. The binding energy between two Mn atoms of course depends on the Fermi level position, but it is always of the order of $1 \mathrm{eV}$; it is therefore large enough to ensure the formation of complexes, provided that $\mathrm{Mn}$ atoms can diffuse.

The formation of $\mathrm{Mn}$ dimers has important consequences on the magnetic properties of Mn-doped silicon. In Table I we show the preferred spin alignment [ferromagnetic (FM) or AFM] of the dimers complexes, along with the charge states, total magnetic moments, and atomic magnetic moments. It was found, in Ge or GaAs, that AFM pairing prevails when $\mathrm{Mn}$ impurities are brought together to form complexes. We find that dimers in silicon, at the considered concentrations, prefer FM spin alignment as ground state configuration, with the only exception of $\mathrm{Mn}_{\mathrm{Si}}-\mathrm{Mn}_{\mathrm{Si}}^{2-/ 4-}$ and $\mathrm{Mn}_{\mathrm{I}}-\mathrm{Mn}_{\mathrm{I}}^{0}$. This finding has important consequences on the magnetic properties of Mn-doped silicon. Indeed, pairing seems not to quench the magnetic properties related to isolated $\mathrm{Mn}$ atoms. In particular, for $E_{F}$ below $0.6 \mathrm{eV}, \mathrm{Mn}_{\mathrm{Si}}-\mathrm{Mn}_{\mathrm{I}}$ dimers-as well as isolated interstitial—are defects with a large magnetic moment, ranging from 2 to 4 Bohr magnetons. For higher $E_{F}$, substitutional pairs are the lowest en-

TABLE I. Relevant information for the considered Mn dimers: charge state ( $Q$, in electrons), favored spin configuration, total magnetic moment $\left(M_{\mathrm{tot}}\right)$, atomic magnetic moments $\left(M_{1}\right.$ and $\left.M_{2}\right)$ in bohr magnetons. In the case of the $\mathrm{Mn}_{\mathrm{Si}}-\mathrm{Mn}_{\mathrm{I}}$ dimer magnetic moments $M_{1}$ and $M_{2}$ refers to the interstitial and substitutional atoms respectively.

\begin{tabular}{lccccc}
\hline \hline & $Q$ & Spin. conf. & $M_{\text {tot }}$ & $M_{1}$ & $M_{2}$ \\
\hline $\mathrm{Mn}_{\mathrm{Si}}-\mathrm{Mn}_{\mathrm{I}}$ & $2-$ & $\mathrm{FM}$ & 4 & 2.33 & 1.75 \\
& $1-$ & $\mathrm{FM}$ & 5 & 2.48 & 1.91 \\
& 0 & $\mathrm{FM}$ & 4 & 2.38 & 1.71 \\
$\mathrm{Mn}_{\mathrm{Si}}-\mathrm{Mn}_{\mathrm{Si}}$ & $4-$ & $\mathrm{AFM}$ & 0 & -1.27 & 1.27 \\
& $2-$ & $\mathrm{AFM}$ & 0 & -1.61 & 1.61 \\
& 0 & $\mathrm{FM}$ & 2 & 0.83 & 0.83 \\
$\mathrm{Mn}_{\mathrm{I}}-\mathrm{Mn}_{\mathrm{I}}$ & $2-$ & $\mathrm{FM}$ & 4 & 2.28 & 2.28 \\
& $1-$ & $\mathrm{FM}$ & 5 & 2.40 & 2.40 \\
& 0 & AFM & 0 & -2.50 & 2.50 \\
& $2+$ & FM & 2 & 1.40 & 1.40 \\
\hline \hline
\end{tabular}


ergy configuration, so that formation of dimers implies transition to an AFM spin alignment; we therefore expect that only $p$-type conditions are favorable for the existence of a magnetic activity in $\mathrm{Mn}$-doped $\mathrm{Si}$, while in $n$-type conditions large Mn concentrations do not necessarily lead to a net magnetization of the material.

So far we focused our attention on the energetics and magnetic properties of $\mathrm{Mn}$ impurities and dimers, considering the Fermi level as an independent variable. This is a reasonable assumption only at very low Mn doping concentrations. Indeed, as shown in Figs. 1 and 2, Mn is not an electrically neutral defect over most of the allowed range for the Fermi level. We find that isolated Mn tend to compensate both $p$ and $n$ doping. Indeed, in $p$-type ( $n$-type) conditions $\mathrm{Mn}_{\mathrm{I}}$ and $\mathrm{Mn}_{\mathrm{Si}}$ are donors (acceptors). As for the dimers, they show a very different behavior. They are neutral defects in $p$-type conditions, and multiple acceptors in $n$-type material. Therefore Mn dimers do not interfere with $p$ doping of Si. This is an important finding, since a ferromagnetic behavior in dilute magnetic alloys requires both the presence of magnetically active dopant and of free carriers. Therefore, we conclude that in $p$-doped Si the effect of clustering, at least in the form of dimers, seems to enhance the magnetic properties of the material.

In summary, our calculations show that in a crystalline silicon host an energy-driven force for $\mathrm{Mn}$ atoms to form complexes exists. We find that the most stable configurations involving up to two $\mathrm{Mn}$ atoms is the $\mathrm{Mn}_{\mathrm{Si}}-\mathrm{Mn}_{\mathrm{I}}\left(\mathrm{Mn}_{\mathrm{Si}}-\mathrm{Mn}_{\mathrm{Si}}\right)$ complex in $p$-type ( $n$-type) conditions. The $\mathrm{Mn}_{\mathrm{Si}}-\mathrm{Mn}_{\mathrm{I}}$ dimer is mostly a neutral defect and shows a FM spin alignment that allows a net magnetization of the material. On the contrary, $\mathrm{Mn}_{\mathrm{Si}}-\mathrm{Mn}_{\mathrm{Si}}$ is an acceptor and shows a preference for AFM alignment in those doping conditions where it is energetically stable. Therefore, we expect that Mn-doped silicon will show magnetic activity only in $p$-type conditions.

This work was supported by the Istituto Nazionale Fisica della Materia (INFM) through Iniziativa Trasversale Calcolo Parallelo.

${ }^{1}$ H. Munekata, H. Ohno, S. von Molnar, A. Segmüller, L. L. Chang, and L. Esaki, Phys. Rev. Lett. 63, 1849 (1989).

${ }^{2}$ A. Haury, A. Wasiela, A. Arnoult, J. Cibert, S. Tatarenko, T. Dietl, and Y. Merlè d'Aubigné, Phys. Rev. Lett. 79, 511 (1997); D. Ferrand, J. Cibert, C. Bourgognon, S. Tatarenko, A. Wasiela, G. Fishman, A. Bonanni, H. Sitter, S. Kolesnik, J. Jaroszyski, A. Barcz, and T. Dietl, J. Cryst. Growth 214/215, 387 (2000).

${ }^{3}$ Y. D. Park, A. T. Hanbicki, S. C. Erwin, C. S. Hellberg, J. M. Sullivan, J. E. Mattson, T. F. Ambrose, A. Wilson, G. Spanos, and B. T. Jonker, Science 295, 651 (2002).

${ }^{4}$ J. Kreissl, W. Gehlhoff, and H. Vollmer, Phys. Rev. B 49, 10307 (1994); J. Martin, J. Wedekind, H. Vollmer, and R. Labusch, ibid. 61, 1918 (2000).

${ }^{5}$ H. Katayama-Yoshida and A. Zunger, Phys. Rev. B 31, 8317 (1985).

${ }^{6}$ F. Beeler, O. K. Andersen, and M. Scheffler, Phys. Rev. B 41, 1603 (1990).

${ }^{7}$ S. B. Zhang and John E. Northrup, Phys. Rev. Lett. 67, 2339 (1991).

${ }^{8}$ J. P. Perdew and Y. Wang, Phys. Rev. B 45, 13244 (1992).

${ }^{9}$ G. Kresse and D. Joubert, Phys. Rev. B 59, 1758 (1999).

${ }^{10}$ G. Kresse and J. Hafner, Phys. Rev. B 47, R558 (1993); G. Kresse, Ph.D. Thesis, Technische Universität Wien, 1993; G. Kresse and J. Furthmüller, Comput. Mater. Sci. 6, 15 (1996); Phys. Rev. B 54, 11169 (1996).

${ }^{11}$ G. Makov, R. Shah, and M. C. Payne, Phys. Rev. B 53, 15513 (1996).

${ }^{12}$ H. Kitagawa, Solid State Phenom. 71, 51 (2000), and references therein. 